\title{
Inverse Leidenfrost Effect: Levitating Drops on Liquid Nitrogen
}

\author{
M. Adda-Bedia, ${ }^{\dagger}$ S. Kumar, ${ }^{\dagger}$ F. Lechenault,${ }^{\dagger}$ S. Moulinet, ${ }^{\dagger}$ M. Schillaci, ${ }^{\dagger}$ and D. \\ Vella*,‡ \\ $\dagger$ †aboratoire de Physique Statistique, Ecole Normale Supérieure; Sorbonne Universités \\ UPMC; CNRS; 24 rue Lhomond, 75005 Paris, France \\ $\ddagger$ Mathematical Institute, Andrew Wiles Building, University of Oxford, Woodstock Rd, \\ Oxford, OX2 6GG, U.K. \\ E-mail: dominic.vella@maths.ox.ac.uk
}

\begin{abstract}
We explore the interaction between a liquid drop (initially at room temperature) and a bath of liquid nitrogen. In this scenario, heat transfer occurs through film-boiling: a nitrogen vapour layer develops that may cause the drop to levitate at the bath surface. We report the phenomenology of this inverse Leidenfrost effect, investigating the effect of the drop size and density by using an aqueous solution of a tungsten salt to vary the drop density. We find that (depending on its size and density) a drop either levitates or instantaneously sinks into the bulk nitrogen. We begin by measuring the duration of the levitation as a function of the radius $R$ and density $\rho_{d}$ of the liquid drop. We find that the levitation time increases roughly linearly with drop radius but depends weakly on the drop density. However, for sufficiently large drops, $R \geq R_{c}\left(\rho_{d}\right)$, the drop sinks instantaneously; levitation does not occur. This sinking of a (relatively) hot droplet induces film-boiling, releasing a stream of vapour bubbles for a well-defined length of
\end{abstract}


time. We study the duration of this immersed-drop bubbling finding similar scalings (but with different prefactors) to the levitating drop case. With these observations, we study the physical factors limiting the levitation and immersed-film-boiling times, proposing a simple model that explains the scalings observed for the duration of these phenomena, as well as the boundary of $\left(R, \rho_{d}\right)$ parameter space that separates them.

\section{Introduction}

When a liquid droplet is deposited onto a hot solid surface the drop may, provided the solid surface is hot enough, levitate on a cushion of its own vapour. This phenomenon was described first by Leidenfrost in the $18^{\text {th }}$ Century $^{1}$ and is therefore known as the Leidenfrost effect. Similarly, the critical substrate temperature above which the 'film-boiling' that makes levitation possible is called the Leidenfrost temperature. In everyday life, the Leidenfrost effect is used to test whether a frying pan is hot enough: flicking a drop of water into the pan will lead to the drop skiting about only if the the pan is above the Leidenfrost temperature of water (around $200^{\circ} \mathrm{C}$ ). Although classical, this intriguing effect has experienced a renaissance of research effort recently with a number of studies focusing on the origin of the film-boiling regime $^{2,3}$ and its relation to surface properties. ${ }^{4}$ Other studies have focused on the possibility of generating motion in this way; ${ }^{5-10}$ indeed the sublimation of dry ice $\left(\mathrm{CO}_{2}\right)$ blocks, the solid analogue of the Leidenfrost effect, has been proposed both as the cause of the eroded gullies that are observed on Mars ${ }^{11}$ and a basic mechanism that could power an engine there. ${ }^{12}$

In these common scenarios, levitation is limited by the time taken for the levitating object to evaporate. An alternative scenario is the "inverse" Leidenfrost effect ${ }^{13}$ in which a liquid droplet is placed on the surface of a bath of a cryogenic liquid with low boiling point, e.g. liquid nitrogen. In this case, the deposited droplet causes the evaporation of the substrate, keeping its own mass constant during levitation. Instead of being limited by the mass of the drop, the duration of the inverse Leidenfrost effect is limited by the heat energy stored within the drop: levitation can only occur while the drop remains hot 
enough to sustain the film boiling of the liquid nitrogen. After levitation ceases, the drop comes into contact with the bath, which (since the density of water is greater than that of liquid nitrogen) usually means that the drop then sinks below the surface, ${ }^{14-19}$ even though floating should be possible for sufficiently small drops. ${ }^{20}$ This phenomenon has been investigated in relation to the film-boiling-mediated heat transfer between two liquids at different temperatures, which occurs in propellant spillage accidents: ${ }^{21}$ if liquid propellant spills accidentally during the test-stand or launching operations of a rocket, a catastrophic explosion (resulting from the detonation of the fuel and oxidizer) is possible. ${ }^{14,21}$

Even though the basic mechanism behind this liquid-on-liquid levitation is essentially the same as that responsible for the usual Leidenfrost effect, our quantitative understanding of it remains only partial. ${ }^{14-18}$ Previous studies focussed on modelling the duration of levitation and its dependence on the radius of the drop. ${ }^{15,16,18}$ However, due to the relative paucity of experimental data, which in any case concentrated on simple liquid drops, ${ }^{14,15,17}$ a complete understanding of the inverse Leidenfrost mechanism is lacking. Furthermore, levitation is only possible for sufficiently small droplets - above a critical size, droplets immediately sink and undergo film-boiling within the bulk. Here, we explore this phenomenon using water based solutions at various densities and droplet radii. We are then able to investigate the conditions that allow for the droplets to levitate as well as the duration of the inverse Leidenfrost effect in each of the floating/levitating and sinking states.

This paper is organised as follows: we begin by giving a more detailed account of our experimental protocol, before giving some raw experimental results. These experiments highlight some discrepancies with previously proposed models and so we then go on to develop a new mathematical model to describe the levitation and film-boiling regimes. This allows us to reassess the experimental data, by making a quantitative comparison between theory and experiment. 


\section{Experimental Methods and Observations}

Our experiments were conducted in a home-made cryostat designed to allow imaging at the boiling temperature of nitrogen. The cryostat is principally built from a styrofoam box (labelled 1 in Fig. 1). To have a side view of the experiment, an opening was cut in one wall and two plexiglass plates glued (one to each side), making a double-paned window (labelled 2 in Fig. 1). To avoid the window frosting a continuous flow of dry air at room temperature was maintained within the gap. The box is closed with a plexiglass plate on its top, keeping a small opening in order to allow gaseous nitrogen to escape.

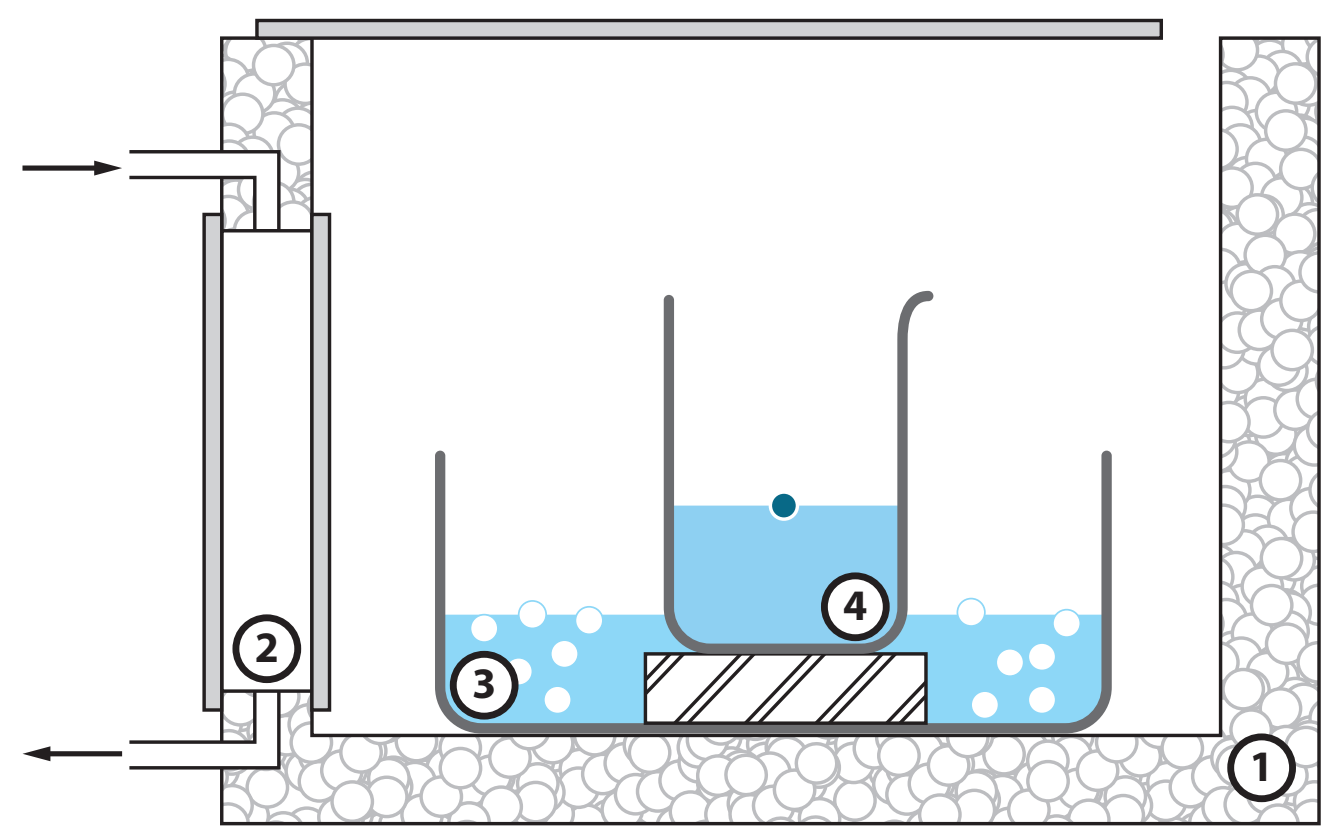

Figure 1: Schematic of the home-made cryostat used in our experiments. The cryostat is built from a $15 \times 20 \times 18 \mathrm{~cm}^{3}$ styrofoam box (1) in which a double-paned window is mounted (2) to allow a side-view of the experiment. To avoid condensation and frosting of this window, a flux of dry air is maintained between the panes. A sacrificial bath (3) sits on the bottom of the cryostat to prevent vigorous boiling of the study bath (4), which is contained in a beaker sitting on an aluminium block within the sacrificial bath.

With the details so far described this cryostat does not insulate the liquid nitrogen well: liquid nitrogen placed inside a beaker boils, creating a rough, bubbling surface. This is not suitable for experiments on the inverse Leidenfrost effect, which require a relatively calm, smooth liquid surface to avoid premature sinking of droplets. To remedy this problem, we 
used two different nitrogen baths: a sacrificial bath (labelled 3 in Fig. 1) contained within a large crystallising dish $(\oslash=14 \mathrm{~cm})$ and sitting on the bottom of the cryostat. The sacrificial bath boils permanently, producing a continuous flow of gaseous nitrogen at its boiling temperature. The cryostat is then completely filled with a thermalized atmosphere of gaseous nitrogen. Within this bath we then place a study bath (labelled 4 in Fig. 1), which is contained in a beaker sitting on an aluminium block. As this bath is surrounded by boiling nitrogen and its vapour, it is well isolated (thermally) and does not spontaneously boil. Its surface is smooth and only evaporates when heated by the proximity of a relatively warm droplet. Within this simple experimental design, we are able to accurately measure the levitation time of a droplet and capture its dynamics (or to measure the film-boiling time for an immersed droplet).

We deliver a droplet to the surface of the study bath using a micropipette. The use of a micropipette allows for droplets of effective spherical radius, $R=(3 V / 4 \pi)^{1 / 3}$ (based on the droplet volume $V$ imposed with an accuracy of $2 \mu \mathrm{l}$ ), in the range $0.5 \mathrm{~mm} \leq R \leq 3 \mathrm{~mm}$. This droplet is initially at room temperature (and hence liquid) but cools and ultimately freezes, becoming opaque in the process (Fig. 2a). In some cases, a droplet breaks into two parts during freezing. We suspect that this behaviour originates from the initial formation of an outer frozen shell, which is broken as the remainder of the drop freezes and, hence, expands. However, the fact that this does not always occur indicates that such an outer shell does not always form, and hence than the heat transfer from the droplet to the study bath is, in general, anisotropic.

If several droplets are deposited on the interface at the same time, they move together and (if this aggregation occurs before the drops freeze) coalesce into a larger drop. We do not study this aggregation here, but attribute it to the interfacial deformations caused by each droplet, which are well known to cause floating bodies to aggregate - the 'Cheerios effect'. ${ }^{22}$ However, even an isolated droplet moves laterally at the surface (see Supplementary Movie); we believe that this motion is caused by small imbalances in the film of vapour on 
which the droplet levitates. We do not study this motion here but conjecture that a small perturbation in one direction leads to a self-perpetuating asymmetry and hence to sustained motion in the direction of the perturbation, analogous to the 'walking droplets' observed on a vibrating liquid bath. ${ }^{23}$
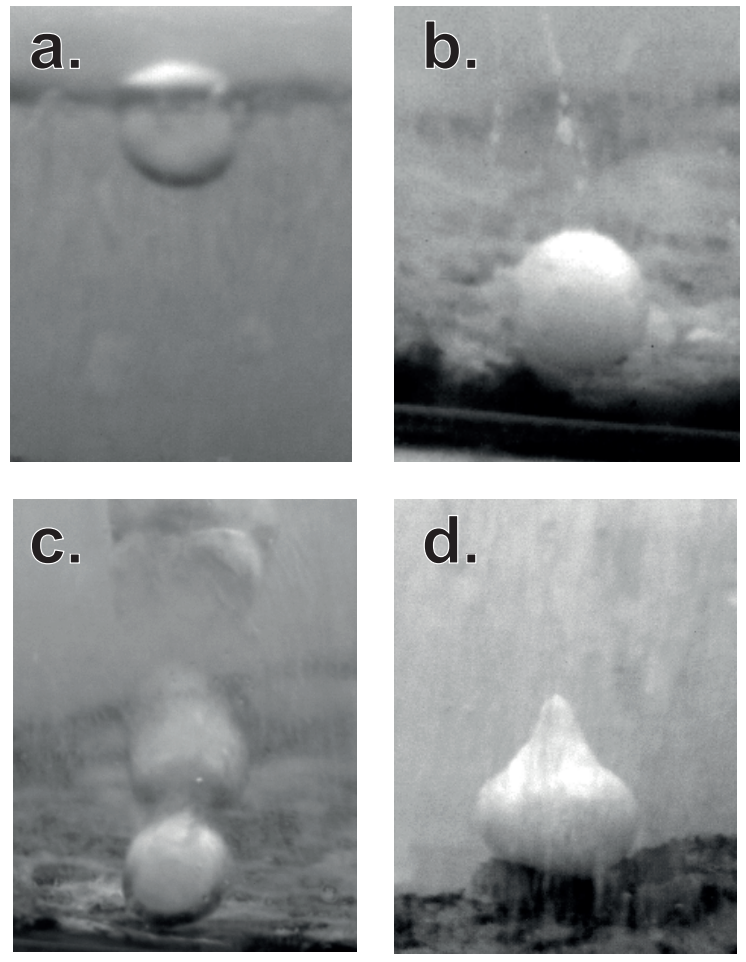

Figure 2: (a) Closeup of a droplet (volume $V=70 \mu \mathrm{l}$, effective radius, $R \approx 2.5 \mathrm{~mm}$ and density $\rho_{d}=1.04 \mathrm{~g} / \mathrm{cm}^{3}$ ) levitating at the surface of the study bath. We see that the droplet remains approximately spherical while levitating. (b) The same drop after sinking is no longer surrounded by a vapour film and the emission of bubbles stops almost immediately. (c) A drop of volume $V=70 \mu \mathrm{l}$, effective radius, $R \approx 2.5 \mathrm{~mm}$ and density $\rho_{d}=1.08 \mathrm{~g} / \mathrm{cm}^{3}$ that does not levitate initially instead sinks to the bottom of the study bath and undergoes film boiling there. Here the drop sits at the bottom of the beaker and emits a string of rising bubbles, which can be seen, albeit slightly blurred by their fast motion, above the drop. (d) When the vapour film disappears, emission of bubbles stops almost immediately revealing that the frozen drop has adopted a 'pear-shaped' form. In both (a) and (c) the drop is surrounded by a vapour film: its apparent thick dark surface is due to internal total reflection on the interface between the liquid and gaseous nitrogen. (See also Movie in Supplementary Material.)

Depending on the size and density of the droplets, the initial levitation reported above may be replaced by instantaneous sinking of the droplet. As well as varying the radius of the droplet (as described above), we therefore varied the droplet density, $\rho_{d}$. To achieve a 
broad range of density variation, we used various dilutions of LST FastFloat (low toxicity sodium heteropolytungstates dissolved in water, supplied by SIGMA ${ }^{\circledR}$ ); this allowed us to create drops with densities in the range $1.02 \mathrm{~g} / \mathrm{cm}^{3} \leq \rho_{d} \leq 2.8 \mathrm{~g} / \mathrm{cm}^{3}$ (undiluted FastFloat). For a given droplet density, levitation is possible only if the radius is below a critical value: above this critical value the droplet sinks into the liquid but remains surrounded by a film of vapour. The immersed drop then provokes vigorous boiling of the surrounding liquid nitrogen. The durations of levitation and this immersed-boiling are, for a given value of drop radius, of the same order of magnitude. In the cases of both levitation and immersed boiling, the droplet ultimately freezes but the final frozen shape of the droplet in each case is quite different: drops that levitate remain roughly spherical (Fig. 2b), while those that sink while undergoing boiling exhibit a pear shape (Fig. 2d). Examination of video footage (Supplementary Material) shows that these drops are spherical when fully liquid (so that the shear stress of the rising bubbles does not significantly deform them). Instead, we believe that the vapour layer is thinner at the base than the top, and hence that cooling occurs more rapidly at the base. In this way the rising bubbles guide the expansion of the droplet

on freezing to form the tip of the 'pear'. While this shape is reminiscent of the cusp-like singularity when a drop sitting on a plane is frozen from below, ${ }^{24}$ our drop has a slightly different form as the heat exchange occurs through a hemispherical surface and the vigorous film-boiling smooths the cusp.

\section{Experimental Results}

Using the experimental apparatus described above, we have investigated the inverse Leidenfrost behaviour of droplets over a large range of radii and densities. Two key questions that we seek to address are: (i) for how long does the inverse Leidenfrost phenomenon persist? and (ii) for which values of radius $R$ and density $\rho_{d}$ does the droplet levitate at the surface of the bath, rather than becoming immersed in the bath? We find that for a given density, 
droplets levitate provided that their radius is below some critical value, $R<R_{c}\left(\rho_{d}\right)$; this is precisely analogous to the surface-tension-supported flotation of small dense objects, which sink above a critical size that depends on their density. ${ }^{20}$ Here we therefore consider first the question of the levitation time of drops for which $R<R_{c}\left(\rho_{d}\right)$ before extending this to the case of immersed droplets, $R>R_{c}\left(\rho_{d}\right)$. Finally, we describe the boundary between levitation and immersion, i.e. we study the form of $R_{c}\left(\rho_{d}\right)$.

\section{Levitation Time}

Our measurements of the time for which a drop is able to levitate (see open circles in Fig. 3) suggest that this time scale is dependent on the drop radius but is relatively insensitive to the density: with almost a factor of three increase in density we see an increase of only a few seconds in the levitation time. However, as already indicated, the maximum radius for which levitation is possible does depend on the density.

\section{Boiling Time}

Drops with a given density, $\rho_{d}$, are only able to levitate if their radius (or equivalently volume) is below a critical value, $R<R_{c}\left(\rho_{d}\right)$. Larger drops instantaneously become immersed in the liquid bath, sinking to the bottom of the study bath. Nevertheless, such drops still exhibit the Leidenfrost phenomenon: liquid nitrogen boils, rising from the drop in a column that destabilizes into bubbles. The measured bubbling time (the time for which the Leidenfrost phenomenon is observed) is shown by the crosses in Fig. 3. The bubbling time of immersed drops is shorter than the levitation time of drops with similar radius (presumably due to the fact that they lose heat over a larger surface area). However, this bubbling time is of the same order of magnitude as the levitation time, and depends on the drop radius in a similar manner. 


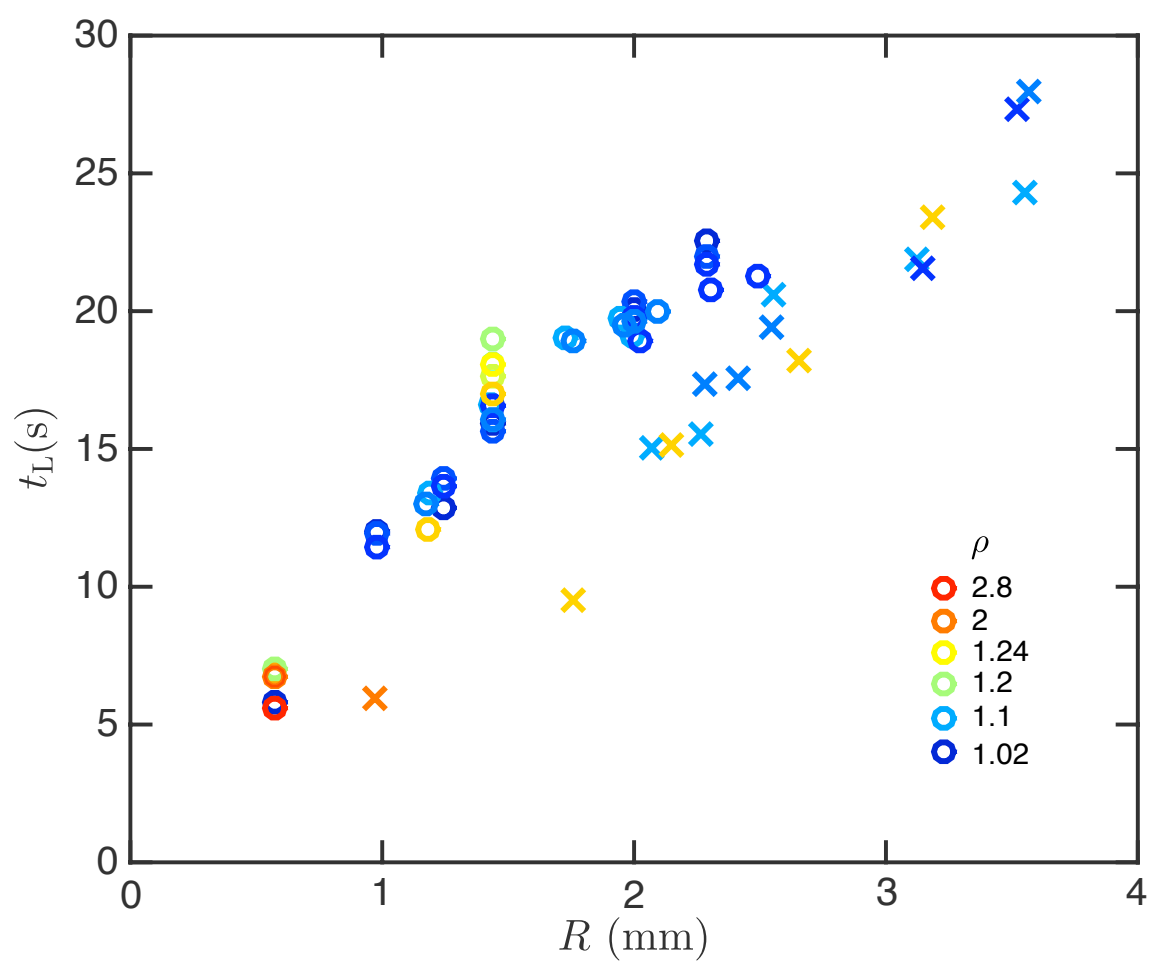

Figure 3: The experimentally measured Leidenfrost time $t_{\mathrm{L}}$ as a function of radius of the drop $R$ for various different drop densities. Each point here is the mean of at least ten repeat experiments and the error bars are smaller than the marker used. Experimental results are shown for both drops levitating at the interface $(\bigcirc)$ and for those that immediately sink, becoming immersed in the Nitrogen bath $(x)$. The density of the droplet is encoded by its colour with red representing neat (undiluted) FastFloat $\left(\rho_{d}=2.8 \mathrm{~g} / \mathrm{cm}^{3}\right)$ and blue the lightest droplets, which have $\rho_{d}=1.02 \mathrm{~g} / \mathrm{cm}^{3}$. Note that the values of $R$ and $\rho_{d}$ correspond to those at room temperature $(300 \mathrm{~K})$, and in the liquid state.

\section{Floating vs Sinking Condition}

Finally, we investigate the regimes of $\left(\rho_{d}, R\right)$ parameter space for which the droplet levitates, or sinks. In the first case, the vapour bubbles produced by the Leidenfrost effect is enough to keep the droplet at the surface. In the second, the bubbles that the drop generates are not enough to lift it back to the surface and it remains immersed at the bottom of the study bath.

Figure 4 presents the experimentally determined regime diagram, and shows the regions of $\left(\rho_{d}, R\right)$-space for which each of these possibilities is observed. We observe that, for a 
given droplet density, there is a sharp levitation-immersed boiling transition at some critical radius, $R_{c}\left(\rho_{d}\right)$. We further note that $R_{c}\left(\rho_{d}\right)$ appears to be a decreasing, continuous function of $\rho_{d}$ : even droplets with a very large density compared to that of the liquid Nitrogen are able to levitate provided they are small enough.

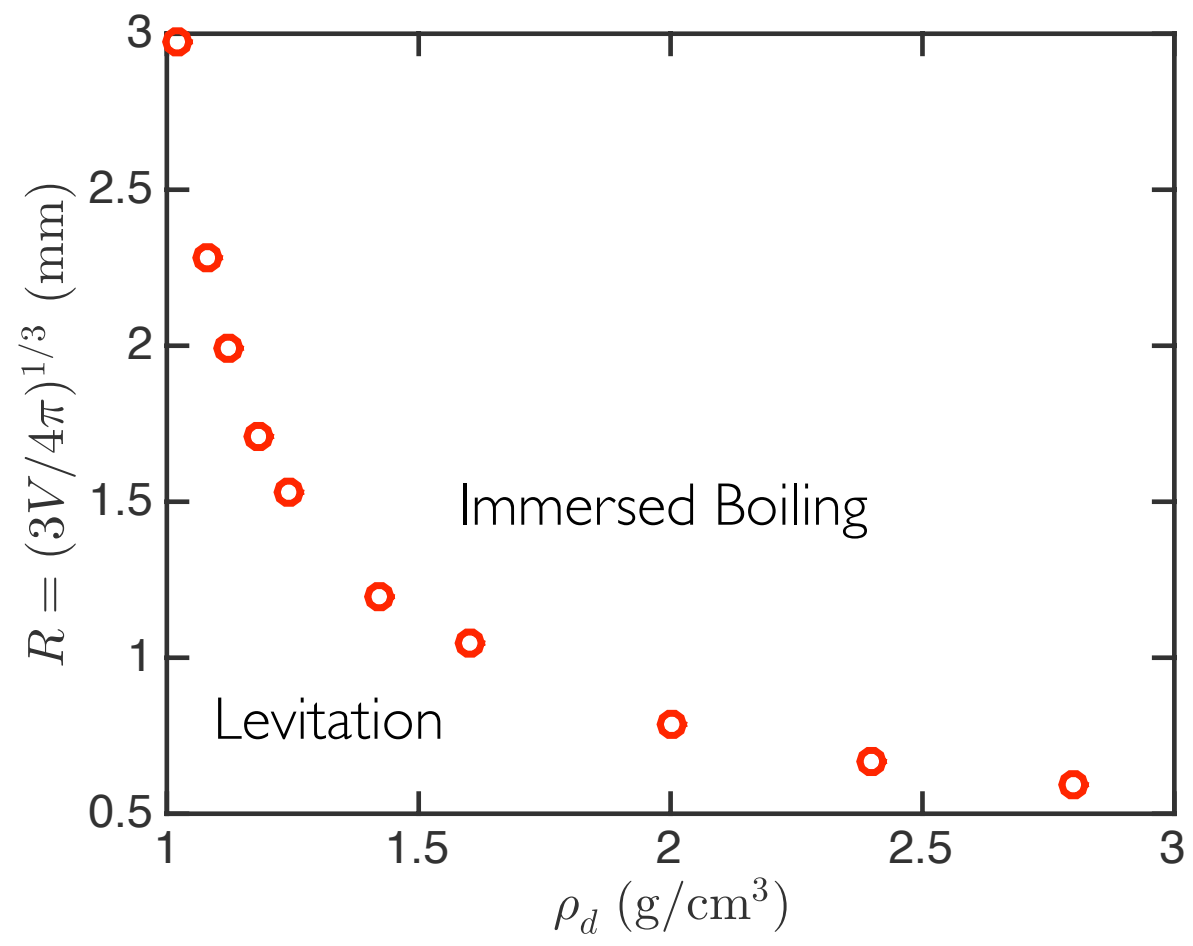

Figure 4: At a fixed droplet density, $\rho_{d}$, only drops below some critical effective radius, $R_{c}\left(\rho_{d}\right)$ will perform the Leidenfrost phenomenon while levitating at the interface. Above this maximum radius, droplets sink into the liquid, becoming fully immersed, and perform the Leidenfrost phenomenon while immersed. Here points show the experimentally determined value of $R_{c}\left(\rho_{d}\right)$ for droplets of different density, $\rho_{d}$, and volumes, $V$, with the effective radius defined to be $R=(3 V / 4 \pi)^{1 / 3}$. All points carry error bars, though these are many times smaller than the actual size of the point on the graph.

\section{Modelling approaches}

Based on our experiments we make three observations of this system that need to be explained quantitatively: the levitation time of droplets (i) varies approximately linearly with the drop radius and (ii) is approximately independent of the droplet density; finally, (iii) 
there is a sharp transition between levitation and immersed bubbling. We first turn to see which, if any, of these observations may be explained by existing models.

\section{Previous modelling approaches}

The mechanisms involved in the inverse Leidenfrost effect have beed described previously. ${ }^{14,15}$ The essential ingredient for levitation is that heat passes from the (relatively warm) drop to the liquid bath beneath it and, in so doing, causes that liquid to evaporate. This leads to the formation of a supporting vapour layer; in this layer, vapour is created and squeezed between the deformed interface and droplet surface eventually escaping into the Nitrogen atmosphere within the cryostat. The pressure field required to perform this squeezing balances the weight of the droplet, allowing it to levitate at the interface. Of course, the droplet contains only a finite amount of thermal energy and, since the evaporation of the liquid Nitrogen requires the supply of latent heat to the bath, the temperature of the droplet decreases until it first freezes itself (releasing latent heat of fusion in the process) before cooling further and ultimately reaches the Leidenfrost temperature of the liquid Nitrogen. At this point, film boiling ceases, the vapour layer disappears and the droplet sinks into the bath.

To quantify the above description requires a model of how the mean vapour layer thickness, $h$, and the temperature of the droplet, $T$, evolve. A key ingredient in this evolution is clearly the heat transfer that occurs both within the droplet and through the vapour layer. Previous detailed studies ${ }^{14,15}$ of the film heat transfer coefficient found it to be $k(1 / R+1 / h)$ with $k$ the thermal conductivity of Nitrogen vapour. It is clear that for a thin layer, $h / R \ll 1$, the $1 / R$ term should be neglected in comparison to the $1 / h$ term (as noted originally ${ }^{14,15}$ ). However, subsequent works ${ }^{16,17}$ retained instead the $1 / R$ term; a scaling analysis shows that this leads to a quadratic dependence of the floating time on the radius of the drop (see the discussion section of this paper), at odds with our experimental results, which suggest something closer to linear. Nevertheless, we note that the levitation times predicted by this model are of the correct order of magnitude according to experiments. ${ }^{14-17}$ As well as 
this confusion over the appropriate heat transfer coefficient, previous studies solved the heat transfer problem (i.e. the evolution of the droplet temperature) under the assumption that the film thickness $h$ does not vary with time. In reality, $h$ evolves as the temperature difference that drives evaporation decreases; in particular, as the temperature difference driving Nitrogen boiling decreases, the film thickness must also decrease to ensure that sufficient hydrodynamic pressure is supplied for the droplet to float. Finally, and because these studies did not consider the vertical force balance on the droplet, there is no previous criterion for determining whether a drop of a given density and radius will initially levitate or become immersed.

\section{Simplifications and assumptions}

The thermal conductivities of water, $k \approx 0.6 \mathrm{~W} / \mathrm{mK}$ and ice $k \approx 2 \mathrm{~W} / \mathrm{mK}$, are significantly larger than that of Nitrogen vapour, $2 \times 10^{-2} \mathrm{~W} / \mathrm{mK} \lesssim k \lesssim 0.1 \mathrm{~W} / \mathrm{mK}$ depending on the temperature. ${ }^{25}$ As a result, while the droplet remains liquid (so that convection may redistribute heat), and even once frozen, we expect the temperature profile within the droplet to remain approximately uniform and thermal conduction across the vapour layer to be rate limiting - this corresponds to assuming that the Biot number (the ratio of conductive flux within the drop to that out of the drop) is small. In this small Biot number limit, the relevant length scale for conduction is only the (unknown) thickness of the thin Leidenfrost layer, $h(\theta, t)$. We further assume that the energy conducted across this gap is immediately converted into Latent heat of vaporization of the gas, and so neglect the thermal profile within the liquid Nitrogen bath. The gas layer around the drop is assumed to be thin so that the flow of the vapour out into the atmosphere may be described by lubrication theory. ${ }^{26}$

In general, the thickness profile (i.e. the spatial variation) of the vapour layer on which the droplet levitates is not known. For the canonical problem of a droplet levitated above a rigid substrate (as in the usual Leidenfrost problem), this problem has been solved in detail ${ }^{27-29}$ and shows that for levitating droplets larger than the capillary length a vapour-filled dimple 
may form beneath the drop, as is observed experimentally. ${ }^{30,31}$ For drops smaller than the capillary length, the bottom of the droplet approximately conforms to the flat substrate. ${ }^{29,31}$ Here, the vapour layer is bounded by two deformable interfaces so that the appropriate capillary length is larger than that of the bare interface: the effective surface tension is larger and the appropriate density is the difference between the droplet and the bath. Our droplets are therefore in the 'small droplet' regime, so that we may simplify the problem (to allow as much analytical progress as possible) by assuming that the thickness of the vapour layer is approximately uniform, i.e. $h(\theta, t)=h(t)$ (i.e. that the drop and bath interfaces conform to one another, as in the small droplet limit of the regular Leidenfrost problem). Furthermore, the surface tension of the drop-vapour interface, $\gamma_{d v} \approx 72.8 \mathrm{mN} / \mathrm{m}$, is significantly larger than that of the bath-vapour interface, $\left.\gamma_{N} \approx 8.85 \mathrm{mN} / \mathrm{m}\right)$. It is therefore energetically favourable for the droplet interface to be deformed as little as possible, at the expense of the bath interface, and so we assume that the shape of the levitating droplet is spherical - in agreement with our experimental observations in Fig. 2a and previous observations of droplets levitating on a vibrated bath (but without phase change). ${ }^{32}$ The simplified problem is illustrated schematically in Fig. 5.

Finally, the physical parameters of the system (e.g. the thermal conductivity $k$ and vapour density $\rho_{g}$ ) vary with temperature. We neglect these variations here, again to facilitate analytical progress. The values of the various parameters that we use here are given in Appendix A; generally we use values for the vapour at a temperature of $200 \mathrm{~K}$, which is roughly midway between the temperature of the study bath $(T=77.36 \mathrm{~K})$ and room temperature. (Choosing a round number for the temperature has the advantage that the thermal properties of Nitrogen gas have been tabulated at intervals of $100 \mathrm{~K} .{ }^{25}$ )

\section{Conservation laws and solution}

Our model for the levitation of a droplet through the inverse Leidenfrost phenomenon is based on the conservation of thermal energy, the conservation of mass (of vapour) and vertical 


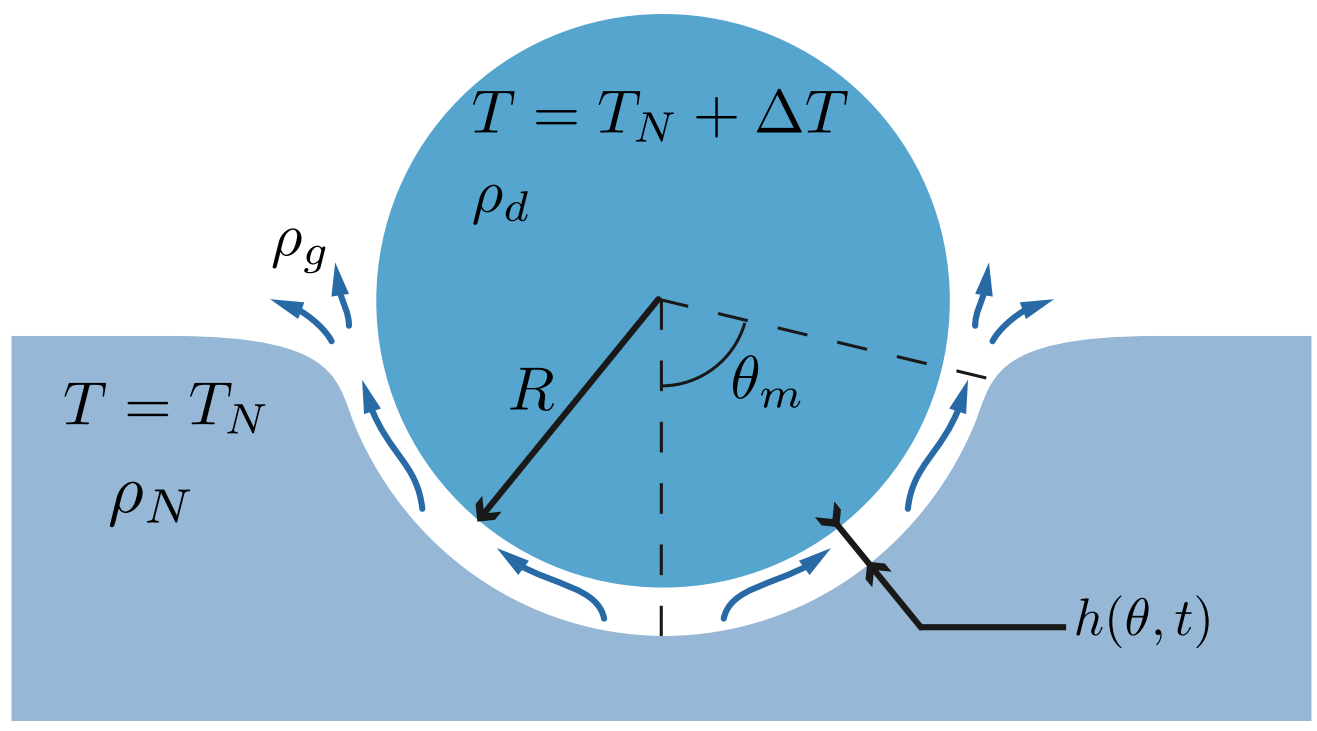

Figure 5: The model setup considered in the theoretical calculations presented here: a spherical droplet sits at the interface of the liquid Nitrogen bath on a cushion of Nitrogen vapour that makes up the Leidenfrost layer. Here we assume that the thickness of this layer is independent of angular position $\theta$, but dependent on time, i.e. $h(\theta, t)=h(t)$.

force balance (on the drop). Since it is the temperature difference between the droplet and the bath, $\Delta T=T_{d}-T_{N}$, that drives the inverse Leidenfrost effect we begin by using conservation of energy to discover how this 'excess temperature' must evolve. The situation is complicated by the fact that the droplet solidifies as it levitates. There are therefore three stages of levitation: in stage I the droplet is liquid and the driving temperature $\Delta T$ evolves; in stage II the droplet freezes, releasing the latent heat of fusion but maintaining a constant $\Delta T$ (assuming conduction of the latent heat is sufficiently fast) and in stage III the droplet is solid and the driving temperature $\Delta T$ evolves once again.

In stage I, the global conservation of energy requires that the rate at which the thermal energy of the (liquid) drop, $\rho_{d} V c_{p} \Delta T$, decreases, must equal the rate at which energy diffuses across the Leidenfrost layer, $k \Delta T / h$, integrated over the surface area, $A$, of the drop that is in close proximity to the Nitrogen bath. We therefore have that

$$
\frac{\mathrm{d}}{\mathrm{d} t}(\Delta T)=-\frac{k A}{\rho_{d} c_{p} V} \frac{\Delta T}{h} .
$$


This is an ordinary differential equation for $\Delta T$ but involves the unknown thickness of the Leidenfrost layer, $h(t)$; an additional equation is required to determine $h(t)$. A similar equation holds for the evolution of the excess temperature in stage III once the drop has solidified; however, the product of the liquid density and heat capacity, $\rho_{d} c_{p}$, is replaced by that of the solid droplet, $\rho_{d}^{(s)} c_{p}^{(s)}$.

In stage II, the freezing of the droplet, it is the latent heat of fusion $\mathcal{L}^{f}$ that must be diffused away over the liquid gap. If we denote by $\phi_{l}$ the proportion of drop that is in the liquid state (so that $\phi_{l}=1$ at the beginning of stage II) then the conservation of energy here reads

$$
\frac{\mathrm{d} \phi_{l}}{\mathrm{~d} t}=-\frac{k A}{\rho_{d} V \mathcal{L}^{f}} \frac{\Delta T}{h} .
$$

In each stage, therefore, we have an expression for the evolution of either the driving temperature difference $\Delta T$ (stages I and III, eqn (1)) or the liquid fraction within the droplet (stage II, eqn (2)). However, each of these evolution equations depends on the (currently unknown) gap thickness $h(t)$. We therefore now turn to determining the evolution of $h(t)$.

We assume that the thickness $h(t)$ is determined by the requirement that the vapour (which is generated by the heat flux from the droplet) be able to drain out of the layer. To proceed, we need to determine the areal flux (a volume per unit time, per unit surface area) $q$ of Nitrogen vapour that is generated by phase change. This flux $q$ is determined by calculating the volume $q$ whose latent heat of vaporization, $\rho_{g} \mathcal{L}^{v} q$, would carry away the instantaneous heat flux, $k \Delta T / h$, across the layer, i.e. $q=k \Delta T /\left(\rho_{g} \mathcal{L}^{v} h\right)$. Since the gap between the droplet and the bath is assumed thin, we then use lubrication theory to describe the motion of the nitrogen vapour within this layer and to determine the pressure field, $p(\theta, t)$, that drives this flow. Combining the standard local conservation of mass equation in lubrication theory ${ }^{26}$ with the source term that comes from the generation of vapour, we have that

$$
\frac{\mathrm{d} h}{\mathrm{~d} t}-\frac{1}{R \sin \theta} \frac{\partial}{\partial \theta}\left(\frac{h^{3} \sin \theta}{12 \mu R} \frac{\partial p}{\partial \theta}\right)=\frac{k \Delta T}{\rho_{g} \mathcal{L}^{v} h}
$$


Equation (3) may be solved, subject to a condition that $p$ remains finite at the base of the drop $(\theta=0)$ and that the pressure is atmospheric at some $\theta=\theta_{m}$, i.e. $p\left(\theta=\theta_{m}\right)=0$. The pressure within the vapour layer is then

$$
p=-\frac{24 \mu R^{2}}{h^{3}}\left(\frac{\mathrm{d} h}{\mathrm{~d} t}-\frac{k \Delta T}{\rho_{g} \mathcal{L}^{v} h}\right) \log \left[\frac{\cos (\theta / 2)}{\cos \left(\theta_{m} / 2\right)}\right] .
$$

Note that the maximum angular position $\theta_{m}$ also enters the conservation of energy (1) or (2) through the area $A$ over which conduction happens.

To progress further, we need to determine from (4) an equation for $\mathrm{d} h / \mathrm{d} t$. This is achieved in different ways for each of the two cases (levitating drops and immersed drops), and so in Appendix B we consider each of these cases separately. However, the result of each calculation is that the evolution equation for the gap thickness may be written in the form

$$
\frac{\mathrm{d} h}{\mathrm{~d} t}-\frac{k \Delta T}{\rho_{g} \mathcal{L}^{v} h}=-c h^{3}
$$

for some dimensional constant $c$ that varies with drop size, density etc.

To make further progress we introduce dimensionless variables

$$
h_{*}=h A / V, \quad \Delta T_{*}=\frac{\rho_{d} c_{p}}{\rho_{g} \mathcal{L}^{v}} \Delta T, \quad t_{*}=\frac{k A^{2}}{\rho_{d} c_{p} V^{2}} t .
$$

Here, the gap thickness is essentially non-dimensionalized by the radius of the sphere, $R$, temperatures by the temperature change of the droplet brought about by changing a unit mass of liquid nitrogen into gas (modified by the density ratio between the gas and the drop) and time is non-dimensionalized by the time scale of thermal conduction over the radius of the drop. In these variables, the evolution equation (1) for stage I of the process becomes

$$
\frac{\mathrm{d}}{\mathrm{d} t_{*}}\left(\Delta T_{*}\right)=-\frac{\Delta T_{*}}{h_{*}}
$$


while for stage II (freezing), we have the dimensionless version of (2)

$$
\frac{\mathrm{d} \phi_{l}}{\mathrm{~d} t_{*}}=-\frac{\rho_{g} \mathcal{L}^{v}}{\rho_{d} \mathcal{L}^{f}} \frac{\Delta T_{*}}{h_{*}}
$$

instead of (7). In stage III, (7) is modified to read

$$
\frac{\mathrm{d}}{\mathrm{d} t_{*}}\left(\Delta T_{*}\right)=-\frac{c_{p}}{c_{p}^{(s)}} \frac{\Delta T_{*}}{h_{*}} .
$$

In each stage, the gap thickness evolves according to the dimensionless version of (5), which may be written

$$
\frac{\mathrm{d}}{\mathrm{d} t_{*}} h_{*}=\frac{\Delta T_{*}}{h_{*}}-C h_{*}^{3}
$$

where the dimensionless constant

$$
C= \begin{cases}\frac{\rho_{d} g V^{5}}{6 \pi \mu R^{4} A^{4}} \frac{\rho_{d} c_{p}}{k}, \quad \text { floating } & \\ \frac{\rho_{N} g V^{4} \rho_{d} c_{p}\left(1-\cos \theta_{m}\right)}{24 k \mu R A^{4} \log \left[1 / \cos \left(\theta_{m} / 2\right)\right]}, \quad \text { immersed. }\end{cases}
$$

Using parameter values typical of our experiments, we find that $C \gg 1$ (typically $10^{5} \lesssim$ $\left.C \lesssim 10^{8}\right)$. In this regime $(10)$ ensures that the thickness of the film evolves approximately quasi-statically, i.e. $h_{*} \approx\left(\Delta T_{*} / C\right)^{1 / 4}$ (see Appendix $\mathrm{C}$ ). The largeness of $C$ ensures that $h \ll 1$, i.e. that the cushioning layer of vapour is only a small fraction of the drop radius, which is a required condition for our use of lubrication theory to be valid.

In this quasi-static limit, we may eliminate $h_{*} \approx\left(\Delta T_{*} / C\right)^{1 / 4}$ from the appropriate form of conservation of energy and solve the resulting equation. For example, from (7) we find that

$$
\Delta T_{*}=\frac{C}{4^{4}}\left(t_{*}^{c}-t_{*}\right)^{4}
$$

and

$$
h_{*}=\left(\frac{\Delta T_{*}}{C}\right)^{1 / 4}=\frac{1}{4}\left(t_{*}^{c}-t_{*}\right) \text {, }
$$


where the constant of integration

$$
t_{*}^{c}=4\left[\Delta T_{*}(0) / C\right]^{1 / 4}
$$

is determined by the initial condition. Physically, the constant $t_{*}^{c}$ corresponds to the (dimensionless) time at which the excess heat of the droplet would vanish if it remained liquid throughout (i.e. in stage I). Of course, the duration of the Leidenfrost phenomenon in this case is more complicated, not least because of the three stages through which the motion passes. We therefore turn now to consider the total duration of the Leidenfrost phenomenon.

\section{Duration of the Leidenfrost phenomenon}

The duration of the first two stages of the motion are relatively simple to calculate since, by definition, stage I ends once the excess temperature $\Delta T$ reaches a critical value $\Delta T_{F}=$

$T_{\text {freeze }}-T_{N}$ (with $T_{\text {freeze }}=-10^{\circ} \mathrm{C}$ the estimated freezing temperature of the polytungstate solution). In dimensionless terms,

$$
t_{*}^{(I)}=\frac{4}{C^{1 / 4}}\left[\Delta T_{*}(0)^{1 / 4}-\Delta T_{F, *}^{1 / 4}\right]
$$

Stage II lasts as long as is required to dissipate the latent heat of fusion. During this stage, the drop remains at $T_{\text {freeze }}$. The dimensionless duration reads

$$
t_{*}^{(I I)}=\frac{1}{C^{1 / 4}} \frac{\rho_{d} \mathcal{L}^{f}}{\rho_{g} \mathcal{L}^{v}} \Delta T_{F, *}^{-3 / 4} .
$$

The final phase of motion is stage III in which the droplet has frozen but continues to cool to the temperature of the bath. We shall assume that this phase ceases when the temperature of the droplet reaches the Leidenfrost temperature of Nitrogen, $T_{c} \approx 126 \mathrm{~K} .{ }^{14}$ 


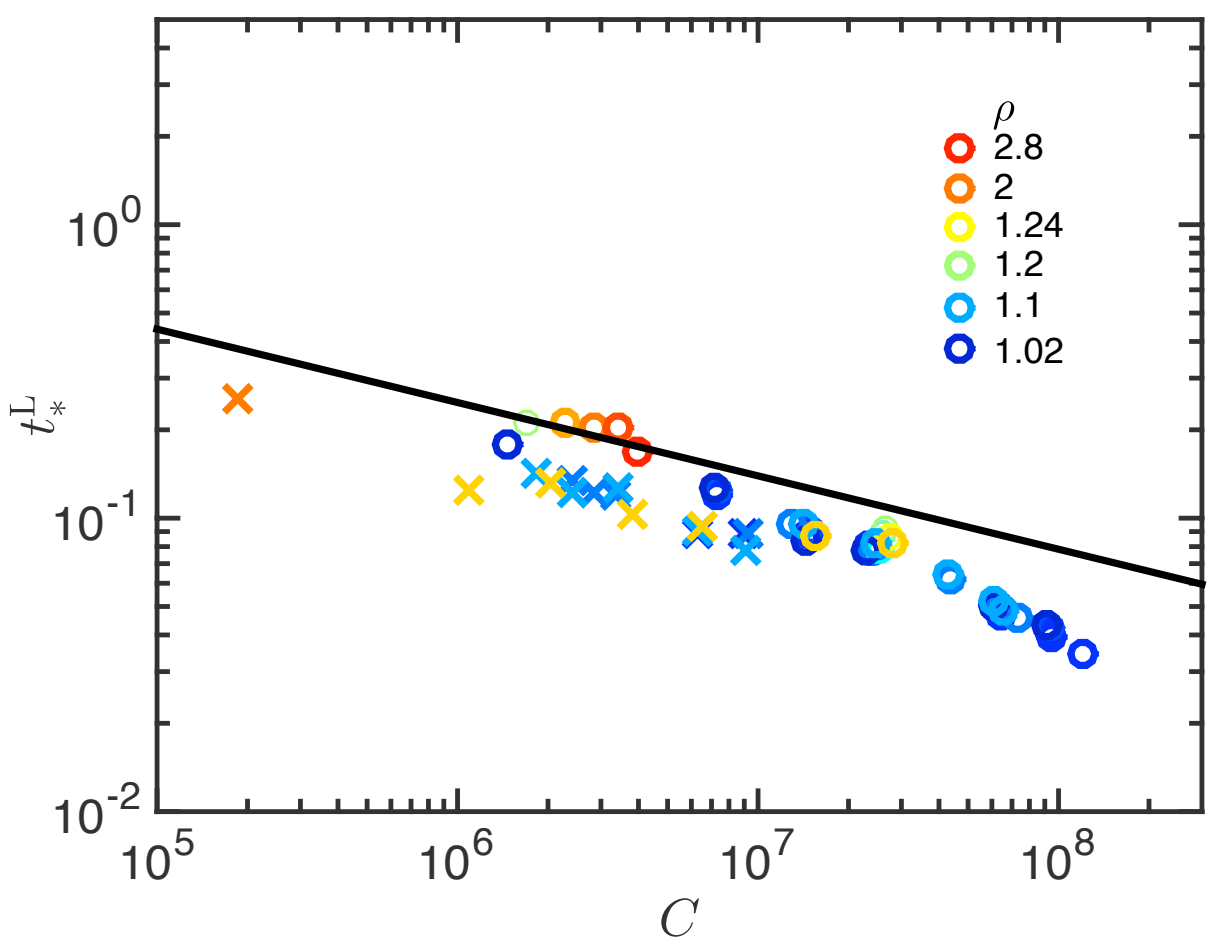

Figure 6: The dimensionless levitation time $t_{*}^{\mathrm{L}}$ plotted as a function of the single dimensionless parameter $C$ given by (11). As in Fig. 3, experimental results are shown for both drops levitating at the interface $(\bigcirc)$ and for those which immediately become immersed in the Nitrogen $(x)$. The density of the droplet is encoded by its colour with red representing neat FastFloat $\left(\rho=2.8 \mathrm{~g} / \mathrm{cm}^{3}\right)$ and blue the lightest droplets (very dilute solutions of FastFloat) for which $\rho=1.02 \mathrm{~g} / \mathrm{cm}^{3}$; a more detailed colour map is given in the legend. The solid line shows the expression (18) obtained from this theory with a final temperature at the end of the Leidenfrost phenomenon of $T_{L}=126 \mathrm{~K} .{ }^{16}$ Note that in evaluating the dimensionless constant $C$ and the theoretical predictions, we have taken the values of $k$ and $\mu$ at $200 \mathrm{~K}$, as detailed in Appendix A.

The dimensionless duration of stage III is thus

$$
t_{*}^{(I I I)}=\frac{4}{C^{1 / 4}} \frac{c_{p}^{(s)}}{c_{p}}\left[\Delta T_{F, *}^{1 / 4}-\left(T_{L}^{*}-T_{N}^{*}\right)^{1 / 4}\right]
$$

and the total dimensionless duration of the Leidenfrost phenomenon is

$$
t_{*}^{\mathrm{L}}=t_{*}^{(I)}+t_{*}^{(I I)}+t_{*}^{(I I I)} \propto C^{-1 / 4} .
$$

The experimentally measured Leidenfrost time (non-dimensionalized as in $(6)$ ), $t_{*}^{\mathrm{L}}$, is 
plotted as a function of the dimensionless parameter $C$ in Fig. 6. This shows two key features of the data: (i) experimental results over a range of size and density (and floating in different states) approximately collapse onto a single curve and (ii) the theoretical prediction in (18) gives a reasonable estimate of the observed Leidenfrost time (recalling that there are no fitting parameters in our model), though the model does over-estimate the duration of the Leidenfrost phenomenon. Furthermore, the experiments suggest a power law behaviour $t_{*}^{\mathrm{L}} \sim C^{-1 / 4}$, as predicted in (18). Finally, given that the duration of each stage of the process scales with $C$ in the same way means that we can estimate the proportions that the drop spends in each stage; we find that the ratios $t^{(I)}: t^{(I I)}: t^{(I I I)} \approx 1: 2: 3$ - each stage occupies a significant fraction of the whole experiment.

In dimensional terms, (18) may be written

$$
\begin{aligned}
t_{\mathrm{L}}=\frac{4}{C^{1 / 4}} \frac{\rho_{d} c_{p} V^{2}}{k A^{2}}\left(\frac{\rho_{d} c_{p}}{\rho_{g} \mathcal{L}^{v}}\right)^{1 / 4}\{ & \left(T_{0}-T_{N}\right)^{1 / 4}-\left(T_{\text {freeze }}-T_{N}\right)^{1 / 4} \\
& +\frac{c_{p}^{(s)}}{c_{p}}\left[\left(T_{\text {freeze }}-T_{N}\right)^{1 / 4}-\left(T_{L}-T_{N}\right)^{1 / 4}\right] \\
& \left.+\frac{\mathcal{L}^{f}}{c_{p}}\left(T_{\text {freeze }}-T_{N}\right)^{-3 / 4}\right\} .
\end{aligned}
$$

Since $V / A \sim R$ and $C \sim R^{3}$, we see immediately that $t_{\mathrm{L}} \sim R^{5 / 4}$, which is almost linear in the droplet radius. Similarly, since almost all occurrences of the drop density $\rho_{d}$ are in combination with the corresponding specific heat capacity $c_{p}$ and if we assume, as discussed in Appendix A, that $\rho_{d} c_{p} \approx$ cst for our solutions, then (19) suggests that the Leidenfrost time is almost independent of the droplet density (with at most a $\rho_{d}^{1 / 4}$ dependence, observed in the levitating regime).

\section{Scaling analysis of Leidenfrost duration}

The detailed theory above shows that the levitation time scales approximately linearly with drop radius, in contrast with most preceding analyses, which suggested a quadratic scaling. 
Furthermore, this time is approximately independent of the drop density. To understand these two surprising observations better, it is worth re-evaluating our model with a brief scaling analysis of the problem. Our key modelling assumption is that the relevant heat flux limiting the cooling of the droplet (and hence the duration of the Leidenfrost phenomenon) is the conduction of heat across the vapour layer, of thickness $h$. We therefore have, in scaling terms, that both the heat flux and the volume flux of vapour into the lubricating layer, $q \sim \Delta T / h$. To determine the gap thickness $h$ we used lubrication theory, which showed that the pressure drop within the lubricating layer $\Delta p \sim \mu u R^{2} / h^{2}$ (see (3)) with $u$ a typical horizontal velocity scale, which must, by mass conservation, be $u \sim q / h$. We therefore have $\Delta p \sim \mu q R^{2} / h^{3} \sim \mu \Delta T R^{2} / h^{4}$. This pressure drop must be equated to either the pressure drop in the external liquid (in the immersed case) or the additional pressure required to support the drop (in the levitating case); either way, we have on purely dimensional grounds that $\Delta p \sim \rho_{*} g R$ (with the value of the appropriate density $\rho_{*}$ depending on the situation) so that $\rho_{*} g R \sim \mu \Delta T R^{2} / h^{4}$ and hence $h \sim\left(\mu \Delta T R / \rho_{*} g\right)^{1 / 4}$. Crucially, this gap thickness is only weakly dependent on the size of the droplet, $R$, and the droplet density $\rho_{d}$. The surface-integrated heat flux out of the droplet, $\sim \Delta T R^{2} / h$, and so the time to lose the initial heat energy of the droplet is $t \sim R h / \Delta T \sim R^{5 / 4} / \rho_{*}^{1 / 4}$, which is approximately linear in the radius $R$ and independent of the droplet density (since $\rho_{d} c_{p}$ is approximately constant for

dilute salt solutions, as discussed in Appendix A). This argument makes it clear that the real root of the quasi-linear dependence of the Leidenfrost time on droplet radius we observe is that thermal conduction happens over the thin cushioning layer, of thickness $h$ that depends only weakly on $R$.

\section{Transition from floating to sinking}

We have now understood the duration of the Leidenfrost phenomenon. However, we have not yet discussed whether a drop of a given density and volume will initially levitate at the interface or will immediately sink, becoming immersed in the liquid and undergoing film- 
boiling. In this section, we seek to understand this question by studying the largest droplet that is able to float for a liquid of a given density. To do this, we draw on the considerable body of work on the 'floatability' of small, dense objects, which has seen intense interest in recent years. ${ }^{20}$

As already assumed in our thermodynamic model, the relatively large tension of the vapour-water interface (relative to that of the liquid-vapour) will ensure that the droplet remains relatively spherical. We therefore treat the Leidenfrost droplet as a perfectly nonwetting $\left(\right.$ contact angle $\left.=180^{\circ}\right)$ sphere and calculate the maximum density a non-wetting sphere of given radius can have for equilibrium flotation to remain possible. This calculation follows closely that presented elsewhere ${ }^{20,33}$ and so here we simply present our numerical results. In this scenario, it is the size of the sphere relative to the capillary length of the interface between the liquid Nitrogen and gaseous Nitrogen, $\ell_{c}=\left(\gamma_{N} / \rho_{N} g\right)^{1 / 2}$, that is important: spheres significantly smaller than the capillary length can be supported at the interface even if their density is larger than that of the supporting liquid. In particular, the maximum floatable radius at a given density is

$$
R \approx \ell_{c}\left(\frac{3 \rho_{N}}{2 \rho_{d}}\right)^{1 / 2}
$$

for $R \ll \ell_{c}$.

Figure 7 shows the data from Fig. 4 replotted in dimensionless terms and compared with the results of this calculation. We see that the experiments do not violate the theoretical upper bound for non-wetting spheres floating at a liquid interface: levitating Leidenfrost drops are no better at floating at the interface than would be a non-wetting sphere of the same density. Indeed we see that drops significantly below the maximum floatable density actually sink, becoming immersed in the liquid Nitrogen bath. One possibility for this discrepancy is that the drops are (by necessity) dropped from a height onto the bath. It has been shown previously that impact at a finite speed may cause the premature sinking 
of solid spheres ${ }^{34}$ and cylinders. ${ }^{35,36}$ Indeed, even if an object is placed at the interface with zero velocity, the acceleration due to gravity in reaching its equilibrium floating depth may induce sinking. ${ }^{35}$

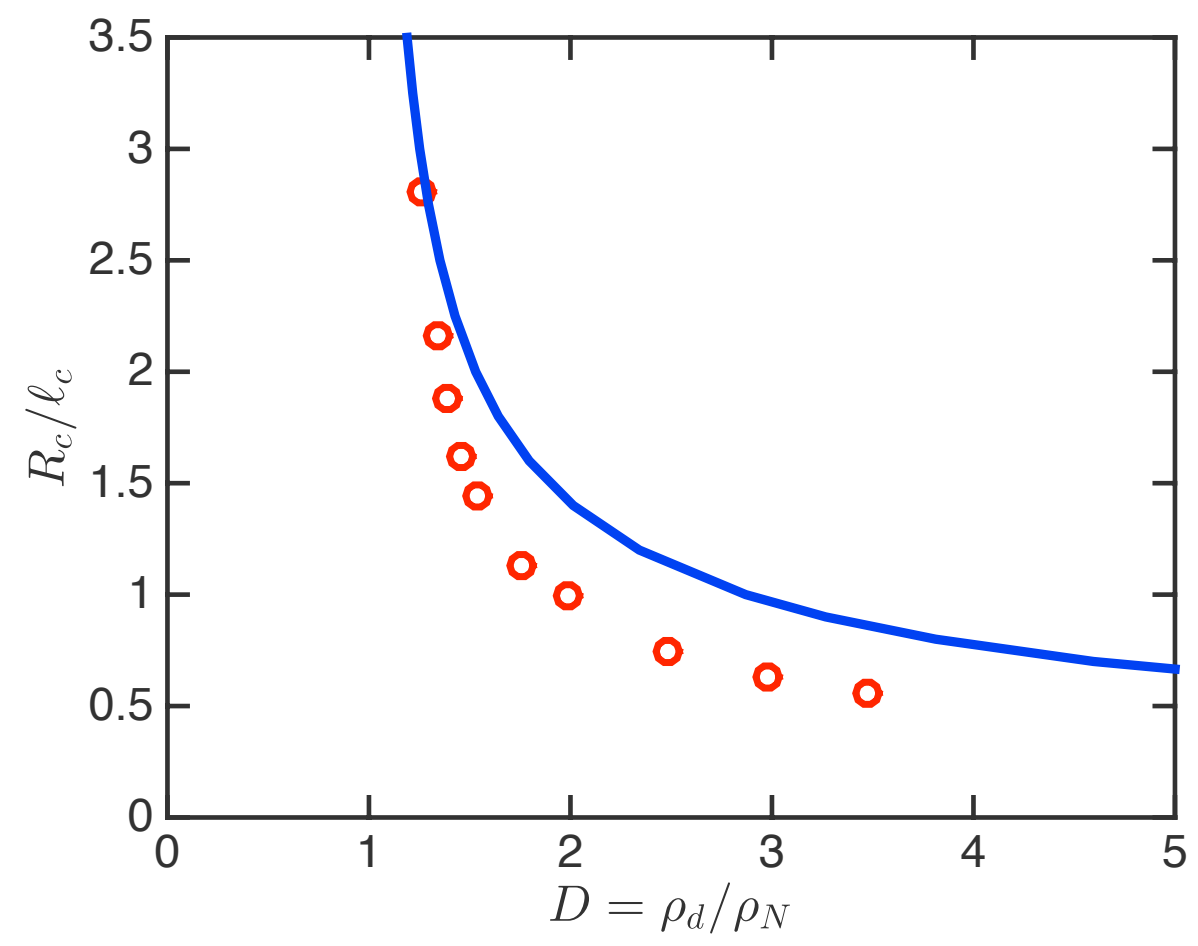

Figure 7: The maximum radius for which levitation is possible, $R_{c}$, as a function of the density ratio, $\rho_{d} / \rho_{N}$, for a perfectly non-wetting sphere is shown by the solid blue curve. The experimentally determined maximum radius for drops of FastFloat at different density are shown by the points and lie below the theoretical maximum. The discrepancy might be attributed to the fact that impact induces premature sinking. ${ }^{20}$

\section{Discussion and Conclusions}

In this paper we have presented a series of experiments on the inverse Leidenfrost effect for a liquid drop on a bath of liquid Nitrogen; these experiments revealed that the levitation time scales approximately linearly with drop radius, in contrast with most preceding analyses, which suggested a quadratic scaling. Furthermore, this time is approximately independent of the drop density. We were able to explain these two experimental observations using 
a theory that coupled heat conduction to the force balance condition required as a result of levitation; the essential ingredients of this theory may be understood from the scaling analysis given above.

While the density of the droplet has little effect on the duration of the Leidenfrost phenomenon, it does play a major role in the phenomenology since, for a given radius, the density of the drop determines whether it floats or sinks. Our experiments suggest that this transition should be understood within the context of the vast literature on the floating/sinking transition of dense interfacial objects, rather than being a particular feature of the Leidenfrost phenomenon.

Looking to the future, we hope that other groups will be motivated to extend our theoretical model to study the effect of spatial variations in the gap thickness $h$, which we have assumed is uniform (but varying in time). This has been done in detail for the regular Leidenfrost problem ${ }^{29,37}$ and for droplets levitating on a vibrating bath in the absence of phase change. ${ }^{32}$ It may also be interesting to investigate the thermal properties of the Polytungstate solutions used here - is our assumption of a constant volumetric specific heat $\rho_{d} c_{p}$ and latent heat $\rho_{d} \mathcal{L}^{v}$ responsible for the discrepancy between experiment and theory observed here? Other intriguing phenomenon worth studying include the self-propulsion of the drops studied here and that of other drops (e.g. Ethanol droplets) that do not sink, even after the Leidenfrost phenomenon has ceased (presumably due to surface tension effects and the smooth surface of frozen Ethanol). This long term continuation of levitation (or, more accurately, floating), may result in a frozen droplet repeatedly bouncing off the edge of the container (see Supplementary Movie). Finally, we note that more violent versions of the Leidenfrost effect are believed to occur when alkali metals are placed at the surface of water and may ultimately be the cause of the explosions that are usually attributed to the hydrogen that is produced via chemical reactions. ${ }^{38}$ 


\section{Acknowledgements}

We thank Dr K. E. Daniels for her support and contributions to this project. This project has received funding from the European Research Council (ERC) under the European Union's Horizon 2020 research and innovation program (grant agreement No. 637334 - GADGET).

\section{Appendix A: Parameter values}

In performing our calculations we have used a range of parameter values from the literature. We summarize these in Table 1 for ease of reference and to aid navigation of the many relevant papers.

For the thermodynamic properties of the polytungstate solutions, we emphasize that the solutions used are prepared at relatively low concentration despite the dramatic increase in density: the concentration of neat FastFloat is approximately $0.6 \mathrm{~mol} / \mathrm{l}$, i.e. a molar fraction of $10^{-2}$. We therefore expect that the molal heat capacity of the solution remains dominated by that of the solvent, water. By neglecting the volume change due to dilution, we adopt the simplifying assumption of a constant volumetric heat capacity: $\rho_{d} c_{p} \approx$ cst. (For more common salts, such as $\mathrm{NaCl}$ and $\mathrm{KCl}, \rho_{d} c_{p}$ varies by less than $5 \%$ for concentrations up to $1 \mathrm{~mol} / 1{ }^{39}$ ) For similar reasons, we assume that $\rho_{d} \mathcal{L}^{f}$ is independent of the concentration.

Table 1: Physical parameter values used in the model.

\begin{tabular}{|c|c|c|c|}
\hline Quantity & Symbol & Value & Ref. \\
\hline Leidenfrost temperature & $T_{L}$ & $126 \mathrm{~K}$ & 16 \\
Viscosity @ 200 K & $\mu$ & $12.89 \mu \mathrm{Pas}$ & 40 \\
Thermal cond. @ 200 K & $k$ & $18.72 \mathrm{~mW} / \mathrm{m} / \mathrm{K}$ & 40 \\
Density of gaseous $\mathrm{N}_{2} @ 200 \mathrm{~K}$ & $\rho_{g}$ & $1.706 \mathrm{~kg} / \mathrm{m}^{3}$ & 41 \\
Latent heat of $\mathrm{N}_{2}$ vaporization & $\mathcal{L}^{v}$ & $2 \times 10^{5} \mathrm{~J} / \mathrm{kg}$ & 42 \\
Density of liquid $\mathrm{N}_{2}$ & $\rho_{N}$ & $807 \mathrm{~kg} / \mathrm{m}^{3}$ & 43 \\
Liquid droplet heat capacity & $\rho_{d} c_{p}$ & $4.2 \mathrm{MJ} / \mathrm{K} / \mathrm{m}^{3}$ & 44 \\
Latent heat of $\mathrm{H}_{2} \mathrm{O}$ fusion & $\mathcal{L}^{f}$ & $334 \mathrm{~kJ} / \mathrm{kg}$ & 45 \\
Solid droplet heat capacity & $\rho_{d}^{(s)} c_{p}^{(s)}$ & $1.9 \mathrm{MJ} / \mathrm{K} / \mathrm{m}^{3}$ & 45 \\
$\mathrm{~N}_{2}$ liquid-vapour tension & $\gamma_{N}$ & $8.85 \mathrm{mN} / \mathrm{m}^{\prime}$ & 46 \\
\hline
\end{tabular}




\section{Appendix B: Details of pressure closure}

In this Appendix, we discuss the remaining condition needed to turn the pressure profile (4) into an equation for the evolution of the gap thickness, $h(t)$. There are two cases to consider: the cases of a levitating and of an immersed droplet.

\section{Floating/levitating}

For a Leidenfrost droplet to levitate at the interface, the vertical component of the pressure force (integrated over the surface of the droplet) must balance the weight of the droplet, i.e.

$$
\rho_{d} g V=2 \pi R^{2} \int_{0}^{\theta_{m}} p(\theta, t) \cos \theta \sin \theta \mathrm{d} \theta .
$$

Substituting for $p(\theta, t)$ from (4) into the above expression and eliminating $\Delta T / h$ in favour of $\mathrm{d} \Delta T / \mathrm{d} t$ using (1) we find that

$$
\frac{\mathrm{d}}{\mathrm{d} t}\left(h+\frac{\rho_{d} c_{p} V}{\rho_{g} \mathcal{L}^{v} A} \Delta T\right)=-c h^{3}
$$

where

$$
c=\frac{\rho_{d} g V}{3 \pi \mu R^{4}\left(3-4 \cos \theta_{m}+\cos 2 \theta_{m}\right)} .
$$

In this case we recover the general form presented in (5).

\section{Immersed}

In the immersed case, there is an unknown reaction force from the base of the tank that balances the weight of the droplet. Instead of the global vertical force balance, therefore, we require that the change in pressure from the bottom of the droplet to the datum of pressure, 
taken at $\theta=\theta_{m}$, should balance the hydrostatic pressure change within the liquid over the same region, $\rho_{N} g R\left(1-\cos \theta_{m}\right)$. We therefore have that

$$
\rho_{N} g R\left(1-\cos \theta_{m}\right)=-\frac{24 \mu R^{2}}{h^{3}}\left(\dot{h}-\frac{k \Delta T}{\rho_{g} \mathcal{L}^{v} h}\right) \log \left[\frac{1}{\cos \left(\theta_{m} / 2\right)}\right]
$$

which can be rewritten in the form of (5) with constant

$$
c=\frac{\rho_{N} g\left(1-\cos \theta_{m}\right)}{24 \mu R \log \left[1 / \cos \left(\theta_{m} / 2\right)\right]}
$$

\section{The choice of $\theta_{m}$}

In both floating and immersed cases, we take $\theta_{m}=\pi / 2$. This value is chosen for simplicity and to represent a reasonable value based on experimental observations: in the immersed case, a thin film is only observed around the lower hemisphere since bubbling becomes violent in the upper hemisphere. Nevertheless, we emphasize that the relative insensitivity of the dimensionless constant $C$ to the parameter $\theta_{m}$ means that the precise value of $\theta_{m}$ used should not be too important.

\section{Appendix C: Nonlinear dynamics of ODEs}

In this Appendix, we briefly discuss the general behaviour of the ordinary differential equations (7)-(10). The key observation is that the dimensionless parameter $C$ is typically large (our experiments generally have $10^{5} \lesssim C \lesssim 10^{8}$ ). In this limit the evolution rapidly converges onto the slow manifold $h_{*} \approx\left(\Delta T_{*} / C\right)^{1 / 4}$ so that the evolution is then quasi-static. This can be illustrated by plotting the trajectories (from the numerical solution of (7) and (10) for stage I of the phenomenon with a range of initial conditions in $\left(\Delta T_{*}, h_{*}\right)$ space), as shown in Fig. 8. 


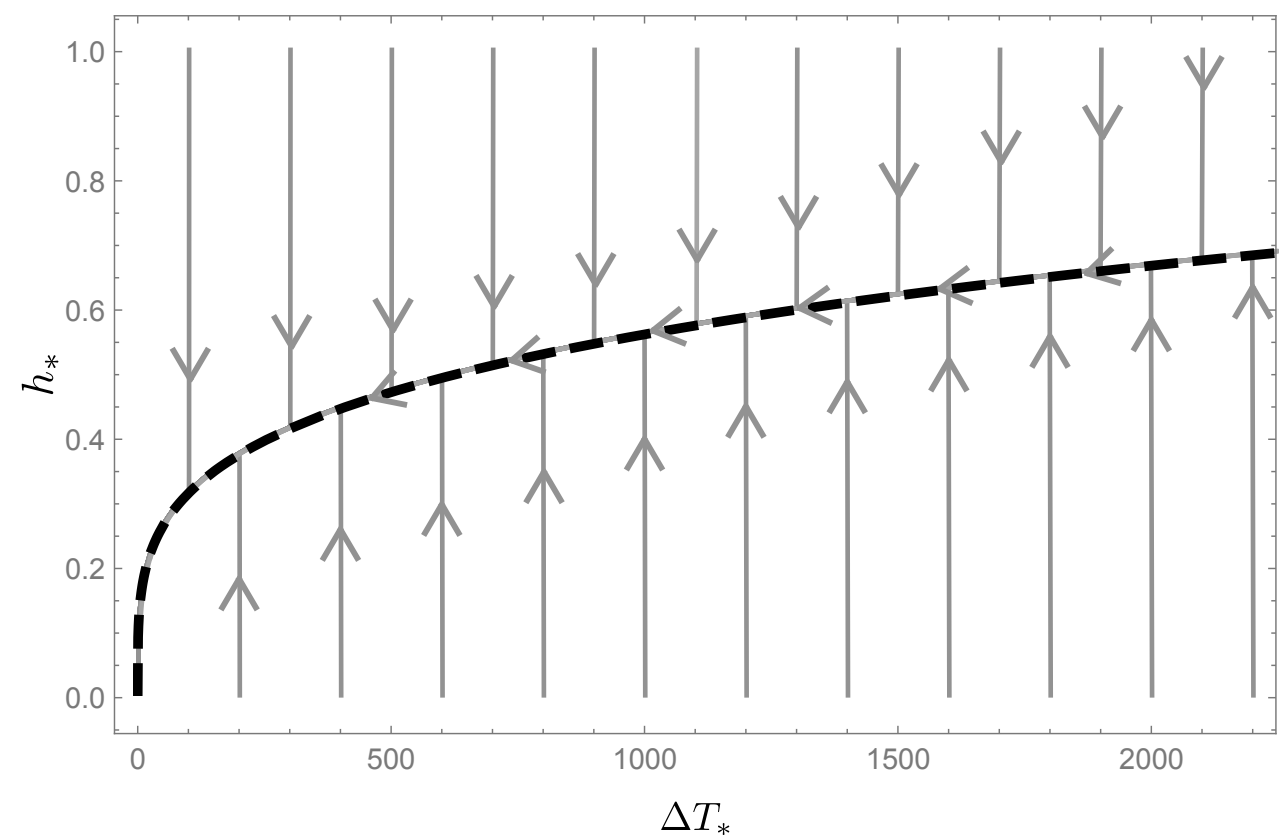

Figure 8: Numerical solution of the full system of ODEs for stage I of the Leidenfrost phenomenon, i.e. (7) and (10) represented in $\left(\Delta T_{*}, h_{*}\right)$ phase space. Each solid curve represents a trajectory for given initial conditions with arrows showing the direction of increasing time. The trajectories are initially very close to vertical showing that the system evolves approximately isothermally until it reaches the quasi-steady state $h_{*}=\left(\Delta T_{*} / C\right)^{1 / 4}$ (dashed curve) and then evolves along this curve. Here $C=10^{5}$ to give results indicative of our experiments.

\section{References}

(1) Leidenfrost, J. De aquae communis nonnullis qualitatibus tractatus; Ovenius, 1756.

(2) Deev, V.; Kharitonov, V.; Kutsenko, K.; Lavrukhin, A. Transient boiling crisis of cryogenic liquids. Int. J. Heat Mass Transf. 2004, 47, 5477-5482.

(3) Lloveras, P.; Salvat-Pujol, F.; Truskinovsky, L.; Vives, E. Boiling Crisis as a Critical Phenomenon. Phys. Rev. Lett. 2012, 108, 215701.

(4) Vakarelski, I.; Patankar, N.; Marston, J.; Chan, D.; Thoroddsen, S. Stabilization of Leidenfrost vapour layer by textured superhydrophobic surfaces. Nature 2012, 489, $274-277$.

(5) Biance, A.; Clanet, C.; Quéré, D. Leidenfrost drops. Phys. Fluids 2003, 15, 1632-1637. 
(6) Quéré, D.; Ajdari, A. Liquid drops: Surfing the hot spot. Nature Mat. 2006, 5, 429-430.

(7) Linke, H.; Alemán, B.; Melling, L.; Taormina, M.; Francis, M.; Dow-Hygelund, C.; Narayanan, V.; Taylor, R.; Stout, A. Self-propelled Leidenfrost droplets. Phys. Rev. Lett. 2006, 96, 154502.

(8) Myers, T.; Charpin, J. A mathematical model of the Leidenfrost effect on an axisymmetric droplet. Phys. Fluids 2009, 21, 063101.

(9) Lagubeau, G.; Le Merrer, M.; Clanet, C.; Quéré, D. Leidenfrost on a ratchet. Nature Phys. 2011, 7, 395-398.

(10) Celestini, F.; Frisch, T.; Pomeau, Y. Take Off of Small Leidenfrost Droplets. Phys. Rev. Lett. 2012, 109, 34501.

(11) Diniega, S.; Hansen, C. J.; McElwaine, J.; Hugenholtz, C. H.; Dundas, C.; McEwen, A.; Bourke, M. A new dry hypothesis for the formation of martian linear gullies. Icarus 2013, 225, 526-537.

(12) Wells, G. G.; Ledsma-Aguilar, R.; McHale, G.; Sefiane, K. A sublimation heat engine. Nature Comm. 2015, 6, 6390.

(13) Hall, R. S.; Board, S. J.; Clare, A. J.; Duffey, R. B.; Playle, T. S.; Poole, D. H. Inverse Leidenfrost Phenomenon. Nature 1969, 224, 266-267.

(14) Hendricks, R. C.; Baumeister, K. J. Heat Transfer and Levitation of a Sphere in Leidenfrost Boiling; 1970.

(15) Hendricks, R. C.; Baumeister, K. J. Liquid or Solid on Liquid in Leidenfrost Film Boiling. Advances in Cryogenic Engineering. New York, 1971; p 455.

(16) Kim, H. Floating phenomenon of a water drop on the surface of liquid nitrogen. J. Kor. Phys. Soc. 2006, 49, L1335-L1338. 
(17) Erickson, D. W.; Williams, G. A. Experimental Studies of the Cryogenic Leidenfrost Effect. Unpublished 2005,

(18) Song, Y. S.; Adler, D.; Xu, F.; Kayaalp, E.; Nureddin, A.; Anchan, R.; Maas, R.; Demirci, U. Vitrification and levitation of a liquid droplet on liquid nitrogen. Proc. Nat. Acad. Sci. 2010, 107, 4596-4600.

(19) Vakarelski, I.; Marston, J.; Chan, D.; Thoroddsen, S. Drag reduction by Leidenfrost vapor layers. Phys. Rev. Lett. 2011, 106, 214501.

(20) Vella, D. Floating Versus Sinking. Annu. Rev. Fluid Mech. 2015, 47, 115-135.

(21) Anon., Propellant Work Shows New Film Boiling Aspect. Chem. Eng. News 1966, 44, 60.

(22) Vella, D.; Mahadvean, L. The 'Cheerios Effect'. Am. J. Phys. 2005, 73, 817-825.

(23) Bush, J. W. M. Pilot-Wave Hydrodynamics. Annu. Rev. Fluid Mech. 2015, 47, 269-292.

(24) Marín, A. G.; Enríquez, O. R.; Brunet, P.; Snoeijer, J. H. Universality of Tip Singularity Formation in Freezing Water Drops. Phys. Rev. Lett. 2014, 113, 05430.

(25) Lemmon, E. W.; Jacobsen, R. T. Viscosity and Thermal Conductivity Equations for Nitrogen, Oxygen, Argon, and Air. Int. J. Thermophys. 2004, 25, 21-69.

(26) Batchelor, G. K. An Introduction to Fluid Dynamics; Cambridge University Press, 1967.

(27) Lister, J. R.; Thompson, A. B.; Perriot, A.; Duchemin, L. Shape and stability of axisymmetric levitated viscous drops. J. Fluid Mech. 2008, 617, 167-185.

(28) Snoeijer, J. H.; Brunet, P.; Eggers, J. Maximum size of drops levitated by an air cushion. Phys. Rev. E 2009, 79, 036307. 
(29) Pomeau, Y.; Le Berre, M.; Celestini, F.; Frisch, T. The Leidenfrost effect: From quasispherical droplets to puddles. C. R. Mecanique 2012, 340, 867-881.

(30) Celestini, F.; Kirstetter, G. Effect of an electric field on a Leidenfrost droplet. Soft Matter 2012, 8, 5992-5995.

(31) Burton, J. C.; Sharpe, A. L.; van der Veen, R. C. A.; Franco, A.; Nagel, S. R. Geometry of the Vapor Layer Under a Leidenfrost Drop. Phys. Rev. Lett. 2012, 109, 074301.

(32) Couder, Y.; Fort, E.; Gautier, C.-H.; Boudaoud, A. From Bouncing to Floating: Noncoalescence of Drops on a Fluid Bath. Phys. Rev. Lett. 2005, 94, 177801.

(33) Vella, D.; Lee, D.-G.; Kim, H.-Y. The load supported by small floating objects. Langmuir 2006, 22, 5979-5981.

(34) Lee, D.-G.; Kim, H.-Y. Impact of a superhydrophobic sphere onto water. Langmuir 2008, 24, 142-145.

(35) Vella, D.; Metcalfe, P. D. Surface tension dominated impact. Phys. Fluids 2007, 19, 072108 .

(36) Vella, D.; Li, J. The impulsive motion of a small cylinder at an interface. Phys. Fluids 2010, 22, 052104.

(37) Maquet, L.; Brandenbourger, M.; Sobac, B.; Biance, A.-L.; Colinet, P.; Dorbolo, S. Leidenfrost drops: Effect of gravity. Europhys. Lett. 2015, 110, 24001.

(38) Fleming, D. Exploding some myths. Educ. Chem. 2015, 14-17.

(39) Horvarth, A. L. Handbook of Aqueous Electrolyte Solutions; Ellis Horwood Limited, Chichester, 1985.

(40) Stephan, K.; Krauss, R.; Laesecke, A. Viscosity and Thermal-Conductivity of Nitrogen for a Wide-Range of Fluid States. J. Phys. Chem. Ref. Data 1987, 16, 993-1023. 
(41) Jacobsen, R. T.; Stewart, R. B. Thermodynamic properties of Nitrogen including liquid and vapor phases from $63 \mathrm{~K}$ to 2000 with pressures to 10000 Bar. J. Phys. Chem. Ref. Data 1973, 2, 757-922.

(42) Dana, L. I. The latent heat of vaporization of liquid oxygen-nitrogen mixtures. Proceedings of the American Academy of Arts and Sciences. 1925; pp 241-267.

(43) Itterbeek, A. V.; Verbeke, O. Density of liquid nitrogen and argon as a function of pressure and temperature. Physica 1960, 26, 931-938.

(44) Ginnings, D. C.; Furukawa, G. T. Heat Capacity Standards for the Range 14 to $1200^{\circ}$ K. J. Am. Chem. Soc. 1953, 75, 522-527.

(45) Handbook of Chemistry and Physics, 66 ${ }^{\text {th }}$ ed.; CRC Press, Inc., Boca Raton, Florida, 1985.

(46) Jasper, J. J. The surface tension of pure liquid compounds. J. Phys. Chem. Ref. Data 1972, 1, 841-1010. 
Graphical TOC Entry

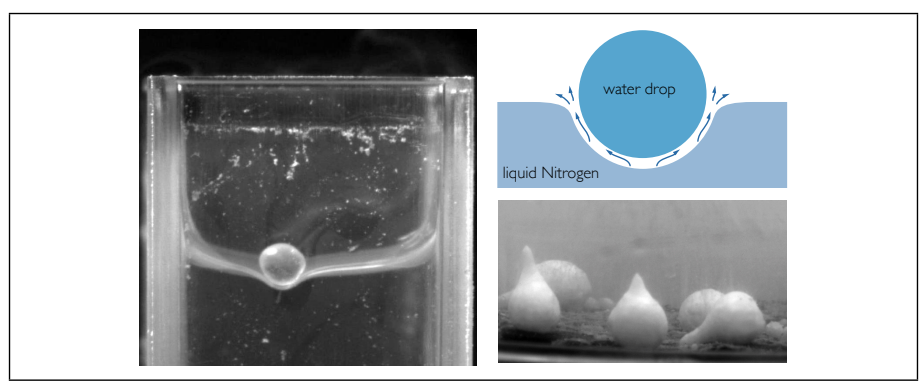

